mo de dicho elemento cede o «tiende a ceder) (de acuerdo con su electronegatividad) en la unidad elemental de esa especie quimica dada. Explicando este concepto y escribiendo las formulas de Lewis de distintas especies quimicas, el educando entiende claramente el concepto de número de oxidación (que no es número de valencia) y lo vincula con la estructura electrónica de la especie química correspondiente. Ello le permite el porqué de cada una de las reglas habitualmente enunciadas para asignar números de oxidación.

El conocimiento de los valores de los números de oxidación de los distintos elementos químicos sobre la base de su ubicación en la tabla periódica, le per* mite luego deducir la formula de un oxoácido mediante un simple cálculo algebraico. Por ejemplo para el ácido sulfúrico:

$$
+\underset{\mathrm{H}_{\mathrm{a}}}{+\mathrm{a}}+\underset{\mathrm{S} \mathrm{O}_{\mathrm{b}}}{6-2 \cdot \mathrm{b}}=\mathrm{O}
$$

Salvo excepciones (que pueden omititse en cursos muy elementales) el valor de $b$ es al menor número que multiplicado por 2 da un valor mayor que 6 (número de oxidación del azufre). Por lo tanto, resulta $b=4 y$, consiguientemente, cebe set $\mathrm{a}=2$. Fórmula obtenjda: $\mathrm{H}_{2} \mathrm{SO}_{4}$.

A partir de las ecuaciones de disociación electrónica total (ecuaciones de ionización total) de los oxoácidos se deducen las fórmulas de los oxoaniones correspondientes.

$Y$ con las fórmulas de los oxoaniones y de los cationes se deducen muy fácil. mente liss fórmulas de las oxosales al equilibrar las cargas de distintos signos.

Concordantemente con estas ideas, en- tre las conclusiones de la Segunda Reunión Nacional de Educación en la Química (REQ-2), efectuada en San Juan, República Argentina, en setiembre de 1984, se incluyen las siguientes recomendaciones:

- desarrollar la enseñanza de las fórmulas químicas de sustancias inorgánicas después de los temas estructura atomica, clasificación periódica, electrone gatividad y uniones químicas.

- deducir las formulas de oxoácidos y oxosales empleando números de oxidación y no valencias, conceptos que no deben confundirse.

BEI.TRAN F.F. Instituto Nacional Superior del Profesorado Técnico Buenos Aires (República Argentina)

\title{
RELACION DE GRUPOS DE TRABAJO
}

GRUPO DE ACTUALIZACION E INVESTIGACION DIDACTICA EN FISICA Y QUIMICA PARA LA E.G.B.

\section{Dirección}

Emigdia Repetto Jiménez y $\mathbf{M}^{\mathrm{a}}$ Carmen Mato Carrodeguas. Profesoras $\Upsilon_{\mathrm{i}}$ tulares de E.U.

\section{Secretaria:}

\section{Lidia Miranda Borges}

\section{Componentes:}

Sebastián Caballero, Pura Eisma, Ma* tilde García, Carmen León, Francisca $M^{a}$ Martínez, Carmen Merillas, Lidia Miranda, $\mathbf{M}^{2}$ Carmen Ruano, Antonio $\mathrm{M}^{2}$ Ruiz y Domingo Suárez. Todos ellos Profesores de E.G.B. en ejercicio.

\section{Dirección del Grupo:}

Escuela Universitaria de Profesorado de E.G.B. (Cátedra de Física y Química). c/ Santa Juana de Arco $s / n$. Las Palmas.

\section{Líneas de trabajo}

Este Gupo se inició en el curso 84-85, con la actualización y perfeccionamiento de los Profesores de E.G.B. que componen el mismo.

Hasta el momento actual ha desarrollado las actividades siguientes:

-Actualización de los profesores adscritos.

- Asesoramiento para el inicio de la Investigación didáctica.

- Revisión de los contenidos progra* mados para el Ciclo Superior.

-Elección de la metodología a seguir para la realización de experiencias pilotos en diferentes niveles de E.G.B. área de Ciencias de la Naturaleza.

-Desarrollo de los Temas elaborados, previa división en equipos de trabajo de los componentes del Grupo, en djversos Centros de E.G.B.

-Evaluación de los resultados

-Valoración de la experiencja.

GRUP ARCOT D'ESTUDIS I DIDACTICA DE LA NATURA

Adreça de contacte:

Joan Cano i Cano

Col+legj Públic Manuel Anton

Partida Barrella. Mutxamel
Comarca I'Alacanti.

\section{Components:}

Antonio Albeza Piqueras, Pere Joan Baeza i Esteve, Antoni Belda i Polo, Joan Josep Cano i Cano (coordinador), Daniel Climent i Giner, Alfred López. i Lara, i Josep Francesc Sogorb i Carratalà.

Linies de treball:

Elaboració i edició de Guies de Camp enfocades al coneixement de distints aspectes de la Natura al País Valencià i dirigides a alumnes del Cicle Superior.

GRUP D'ESTUDIS I DIDACTICA DE I.A NATURA «CADEC»

Nova adreça:

C/ Regidor Ocana, 23

Alacant 03013, Tl. 250789

Components:

Danie! Climent i Giner, Rosa Martín Martinez, Fernando Ballenilla García, Vincent Sansano i Belso, Esperança Valero i Macià, Ferran Ramon i Boix. 
SEMINARIO PERMANENTE DE FISICA Y QUIMICA «VEGAS ALTAS DEL GUADLANA"

El Seminario Permanente de Fisica y Quimica «Vegas Altas del Guadiana» surgió en el curso 1983/84 como iniciativa de un grupo de profesores de Físjca y Química de la zona (radio de 40 $\mathrm{kms}$ ), en respuesta a una preocupación por la mejora de la enseñanza de la Fisica y la Química.

La idea fundamental que inspiró este Seminario Permanente fue el cambio en la metodologia de la ensentanza de la Fí. sica y la Química. Como primer trabajo abordamos un cambio en la enseñanza de la Quimica de COU: "Método activo en la Química de COUn, que fue subvencionado por la Subdirección $\mathrm{Ge}$ neral de Perfeccionamiento del Profesorado, para lo que en el primer elaboramos unos apuntes de Química, que se ajusten a los contenidos del temario del ICE de la Universidad de Extremadura y un cuaderno de prácticas. Con este método pretendemos:

a) Reducir al mínimo las lecciones magistrales.

b) Participación activa del alumnado. Se comienza el tema con una lectura del mismo, realizando, a continuación, las prácticas de laboratorio programadas, los ejercicios numéricos y las actividades bibliográficas propuestas, trabajándose en grupos y sometido todo a una puesta en común final.

c) Mejorar la motivación, interés y rendimiento respecto a la enseñanza tradicional.

Proyectos: a) A lo largo del curso $1984 / 85$ se evaluarán los resultados de la experiencia, siendo reprogramados todos los aspec. tos que sean deficientes. Asímismo, se estudiará la viabilidad de su aplicación en otros Centros.

b) Revisión conceptual y metodológica de la Física y la Química en el BUP, pues el curriculum actual lo considera* mos repetitivo $y$, en algunos casos, absoluto.

\section{Componenies:}

Lourdes Caballero Donoso, Tomás Caballero Rodriguez, Angel Coballes Rius, Joaquin Espinosa Garcia, José B. González Sánchez, Fernando Granel Sánchez, Rosa A. Marqués Queimadelos, Miguel Montero Pilar, Carlos Novillo-Fertrell y Vitrián, José Mel. Ramírez Fernánez, Tomás Román Ga. lán, Silverio Vega Fernández, Félix Vtvas Ramos, Juan Zambrano Morán.

\section{Dirección:}

Seminario Permanente de Física y Qufmica.

"Vegas Altas del Guadiana»

I.B. «Luis Chamizo"

Don Berito-Villanueva de la Serena (Badajoz)

GRUPO DE ENSEÑANZA DEL INSTITUTO DE FISICA DE LA UNIVERSIDAD FEDERAL DE RIO GRANDE DEL SUR

Dirección:

Instituto de Física - UFRGS

90000 Porto Alegre, RS - BrasiI
Fono: (0512) 217666

\section{Componentes:}

Marco Antonio Moreira, (Ph. D. Enset̃anza de Ciencias; coordinador), Rolando Axt (M.Sc. Enseffanza de Física), Bernardo Buchweitz (Ph. D. Ensentanza de Ciencias), Victor Hugo Guimarāes (Especjalización Ensentanza de Física), Carlos E. Levandowski (Ph. D Enseñanza de Ciencias), Fernando Lang da Silveira (M. Sc. Enseñanza de Física), Beatriz Zawisiak (M. Sc. Eduación).

\section{Lineas de Trabajo:}

Enseñanza de laboratorio de Física: producción y testaje de nuevos conjuntos de experimentos; elaboración y testaje de nuevos manuales de laboratorio; evaluación de curriculos de laboratorios de Física fundamentada en un análisis epistemológico de la estructura de experimentos de laboratorio.

Aprendizaje de conceptos físicos: análisis del aprendizaje de conceptos físicos con base en la teoria de $\mathrm{Da}$ vid Ausubel, mapeamiento de contenido y mapeamiento cognoscitivo, según diversas técnicas; mapas conceptuales en la enset̀anza, en la evaluación del aprendizaje y en el análisis de currículo; detección de conceptos espontáneos a través de entrevistas clfnicas.

Formación de recursos humanos en enseñanza de Física: cursos de extensión y perfeccionamiento para profesores de cursos de ciencias; curso de postgraduación (M. Sc. en Física - área de concentración enseñanza) para profesores universitarios.

\section{PRESENTACION DE LINEAS DE TRABAJO}

UN METODO ASEOUIBLE PARA INTRODUCIR A LOS ESTUDIANTES EN EL DOMINIO DE LA HIS. TORIA DE LAS CIENCIAS.

Hemos comprobado con estudiantes de $3^{\circ}$ de BUP, COU y primer ciclo de ca. rreras universitarias que existe un mé. todo fácil y asequible para introducirs los en el dominio de la Hisioria de las Ciencias. El método consiste en aprovechar efemérides de acontecimientos históricos que puedan motivarlos para realizar trabajos de «investigación histórican con los documentos fácilmen. te accesibles - generalmente, secun- darios - que se encuentran en las bibliotecas de Institutos de Bachillerato, Colegios, Universidades e instituciones públicas o privadas.

El procedimiento consta de las siguientes fases:

1. Motivación de los alumnos. 